\title{
Ionization processes in the atmosphere of Titan
}

\section{Electron precipitation along magnetic field lines}

\author{
G. Gronoff ${ }^{1}$, J. Lilensten ${ }^{1}$, and R. Modolo ${ }^{2}$ \\ ${ }^{1}$ Laboratoire de Planétologie de Grenoble, Université Joseph Fourier - CNRS, France \\ e-mail: guillaume.gronoff@obs.ujf-grenoble.fr \\ ${ }^{2}$ LATMOS/IPSL/UVSQ,10-12 avenue de l'Europe, 78140 Vélizy, France
}

Received 20 March 2009 / Accepted 13 July 2009

\begin{abstract}
Context. The Cassini probe regularly passes the vicinity of Titan, providing new insights into particle precipitation by use of its electron and ion spectrometers. A discrepancy between precipitation models and observations of electron fluxes has been found. This discrepancy was suspected to be caused by the geometry of the magnetic field.

Aims. In this article, we compute the electron impact ionization in the nightside ionosphere of Titan, assuming non-trivial geometry for the magnetic field lines.

Methods. We use the TransTitan model, modified to take into account the magnetic field line geometry in the nightside, and we compare these results with the electron flux measurements during the T5 fly-by of Cassini. We use several magnetic field line geometries, including one produced by hybrid simulations.

Results. The geometry of the lines implies a longer path of the electron inside the atmosphere of Titan. The electron fluxes are therefore modified considerably compared to the vertical precipitation hypothesis. At an altitude of $1200 \mathrm{~km}$, the electron flux can be divided up to ten times with a field line resulting from hybrid simulation. Thanks to the use of more accurate field lines, the model reproduces the experiment well without any further adjustment of the precipitated measured electron flux.

Conclusions. Several hypothesis had been suggested to explain the discrepancies between the different models and the observation of the electron flux during the T5 fly-by of Cassini. Our approach shows that the most probable explanation is the magnetic field line geometry. This work shows that the computation of ion production by electron impact in the atmosphere of Titan needs the consideration of both magnetic field and the input electron fluxes. Based on these considerations, our model can compute the conditions for future fly-by, and could be used to compare models with experiments.
\end{abstract}

Key words. planets and satellites: individual: Titan - atmospheric effects - space vehicles: instruments

\section{Introduction}

The magnetic environment of Titan in the magnetosphere of Saturn is very complex, and its characterization and comprehension is one of the main objectives of the Cassini's probe mission (Blanc et al. 2005). Because of the absence of an intrinsic magnetic field, Titan's atmosphere and ionosphere interact directly with Saturn's magnetospheric plasma (Backes et al. 2005). The upper atmosphere is thus partly ionized by mainly solar photons and electron impact. The strength and location of each ionization source is sensitive to the orbital location of Titan, leading to a very complex ionized environment and being highly dynamic. Cassini observations have suggested that photoionisation is the most important ionization process for Titan's dayside (Agren 2009, submitted to PSS). On the other hand, Cassini observations have also shown that, on the nightside, electron impact plays an important role and is the main source of ionization (when the nightside corresponds to the ram-side). This situation happened on April 16th, 2005 on the outbound pass of the T5 flyby by the Cassini spacecraft. The electron flux data acquired during this flyby was compared with several models (Agren et al. 2007; Cravens et al. 2008). These comparisons showed that the ionization and the fluxes detected by the Cassini probe cannot be modeled as a vertical precipitation. This can be interpreted as a consequence of the interaction between the mag- netic field lines and the atmosphere of Titan (Cravens et al. 2009; see also, Gronoff et al. 2009, Paper I - Ionization in the whole atmosphere, this issue). In this paper, we use the TransTitan model to compute the electrons fluxes at different altitude by considering different geometry of magnetic lines.

\section{The TransTitan model}

To compute the electron fluxes in different geometric configurations, we need a code capable of computing electron transport in the atmosphere. In particular, it must compute the influence of elastic and inelastic collisions with the different species of the atmosphere. This code exists: TransTitan, which has been studied in details in the Paper I. In the present paper, we only use the kinetic part for the electrons in the nightside. The geometry of the code is modified to take into account the bending of the magnetic field lines around Titan: in the original version of the code, the electron transport was computed along a straight line, which cannot be strongly inclined with respect to the vertical. The vertical line represents the grid where the ionization is computed. In the present version, we compute the ionization along the magnetic field line. For this study, the neutral atmosphere used is described in Cui et al. (2009), and we take into account only the densities of the two major components of the atmosphere, $\mathrm{N}_{2}$ and $\mathrm{CH}_{4}$. To study the influence of the neutral atmosphere, 


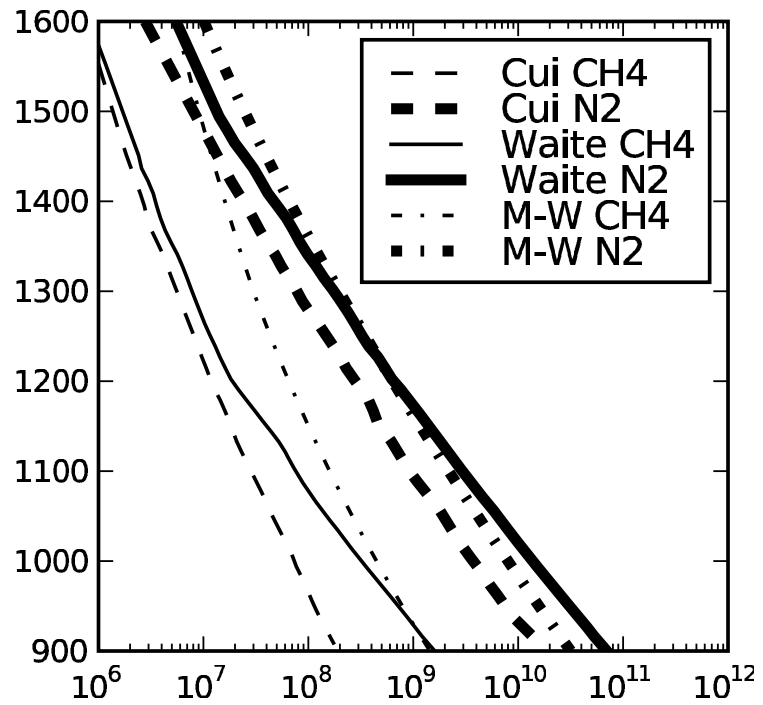

Fig. 1. The different neutral atmosphere considered. For the main simulation, the Cui et al. (2009) neutral atmosphere, coming from Cassini observations, is used. It has a lower density in $\mathrm{CH}_{4}$ and $\mathrm{N}_{2}$ than the Waite et al. (2005) neutral atmosphere (Cassini observations). The preCassini model of Müller-Wodarg et al. (2000) (labeled M-W in the figure) is mainly at a higher density for the two species, the $\mathrm{CH}_{4}$ mixing ration was supposed to be more important than the recent observations.

we also used the neutral atmosphere of Waite et al. (2005) and the pre-Cassini model of Müller-Wodarg et al. (2000). The differences between these models can be seen in Fig. 1. The main difference is that the pre-Cassini model has a higher $\mathrm{CH}_{4}$ density in the upper atmosphere. We assume that the exospheric temperature is constant at $175 \mathrm{~K}$. The altitude range covered by our computation is from $900 \mathrm{~km}$ to $1600 \mathrm{~km}$, the lower altitude being determined by the geometry of the line. The corresponding length of the lines can be more than $4000 \mathrm{~km}$.

\section{Electron precipitation and magnetic field line geometry}

In situ electron-flux spectra are taken from Cravens et al. (2009). The aim of this present work is to compute the ionospheric parameters, by taking into account the presence of the Kronian magnetic field and mimicking the behavior of the electron fluxes, which are affected by the magnetic field draping. So far, vertical magnetic field lines or parabolic lines have been used to compute the electron energy deposition in Titan's atmosphere (Cravens et al. 2005). We assume several magnetic field line configurations, including horizontal magnetic field lines above $1200 \mathrm{~km}$. Titan's ionosphere acts as an obstacle and modifies the magnetospheric plasma flow. The kronian magnetic field lines close to Titan are twisted and wrap the ram-side of the body, as they diffuse slowly in the dense and cold ionosphere. We assume curved magnetic field lines, which enter inside the ionosphere to emerge outside several thousand of kilometer apart. To demonstrate how the magnetospheric electron fluxes are sensitive to the magnetic field line topology, we use simulation results describing the draping around Titan. These field lines are extracted from a threedimensional multi-species hybrid simulation. In this model, a kinetic description is used to describe ions, while electrons are characterized as an inertialess fluid contributing to electric currents that produce quasineutrality. This model depicts the dynamics of the plasma environment in the vicinity of Titan, and includes the effects of ionization by solar photons, ionization by

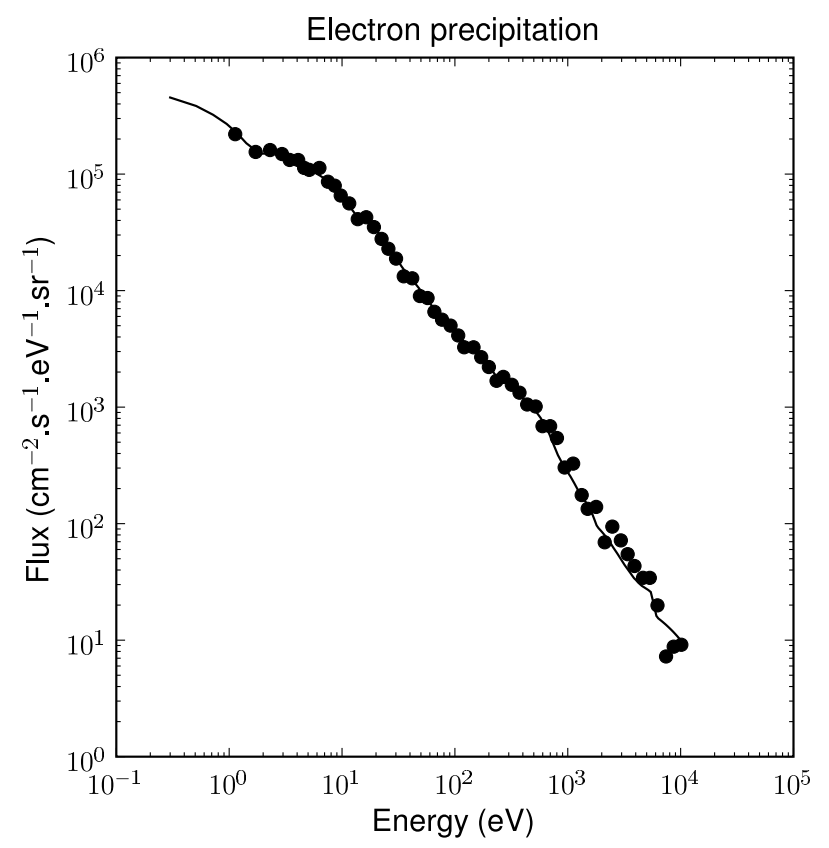

Fig. 2. Electron precipitation flux in the atmosphere of Titan as measured by Cassini ELS instrument, at an altitude of $2730 \mathrm{~km}$ (Cravens et al. 2009). The dots represents the measurement, the line represents the values used in our model. An interpolation has been made between 0.3 and $1 \mathrm{eV}$.

electron impact, and charge exchange. We note that the description below $1400 \mathrm{~km}$ is limited in this model since the complex ionospheric chemistry is not included. Nevertheless, the model describes the photoabsorption and the deceleration of ions in the atmosphere caused by elastic collisions. More details concerning the model are presented in Modolo \& Chanteur (2008). The different configurations are summarized in Fig. 3, where each number corresponds to the following cases:

1. the vertical case;

2. a straight line with a minimum altitude at $1200 \mathrm{~km}$;

3. a straight line with a minimum altitude at $1000 \mathrm{~km}$;

4. a bent line with a minimum altitude at $1200 \mathrm{~km}$;

5. a bent line with a minimum altitude at $1000 \mathrm{~km}$;

6. the hybrid simulated line. This line is cut at the lowest altitude of $1000 \mathrm{~km}$ because the simulation is not valid below. To show the draping at the origin of this line, we put two other simulated lines (label 7), which does not reach the altitude of our simulation.

\section{Result}

Given the incident precipitation flux shown in Fig. 2, we considered the impact of several magnetic geometries on the production and fluxes at different altitudes.

\subsection{First case: vertical precipitation}

This is the simplest case, we consider that electrons simply precipitate vertically. The resulting ionization can be seen in Fig. 4. The ionization peak is at $800 \mathrm{~km}$, and has an intensity of $3 \mathrm{~cm}^{-3} \mathrm{~s}^{-1}$, this simulation having been completed using the Müller-Wodarg et al. (2000) neutral atmosphere, because electrons reaches the mesosphere. We computed the resulting electron flux (which corresponds to the degradation in energy 
90

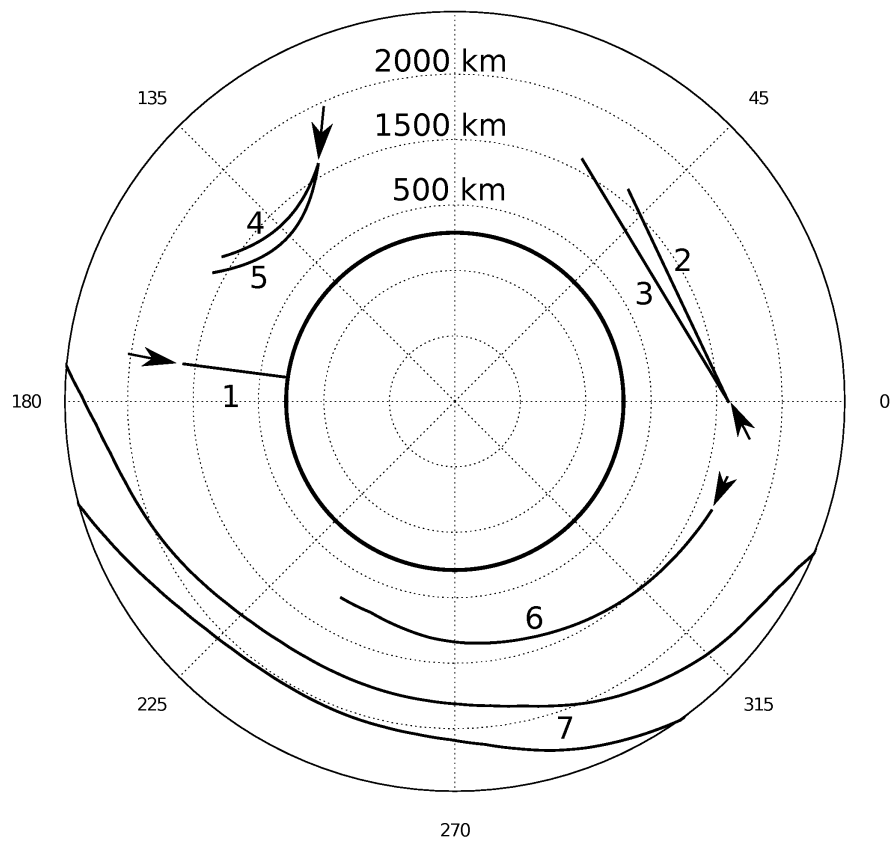

Fig. 3. Magnetic field line geometry used in the simulations. The two straight lines have a minimum altitude of 1200 and $1000 \mathrm{~km}$ respectively, as is also true for the two bent lines. The long line corresponds to hybrid simulations. The angle corresponds to a curvilinear projection: the length on the picture corresponds to the line length, this projection being useful when solar source is not taken into account, as in this paper. The arrows show where the electrons are precipitating in our model, and the large black numbers indicate the simulation number (see the different cases) corresponding to each line. The number 7 shows additional lines from the hybrid simulation, above the altitude of the present study.

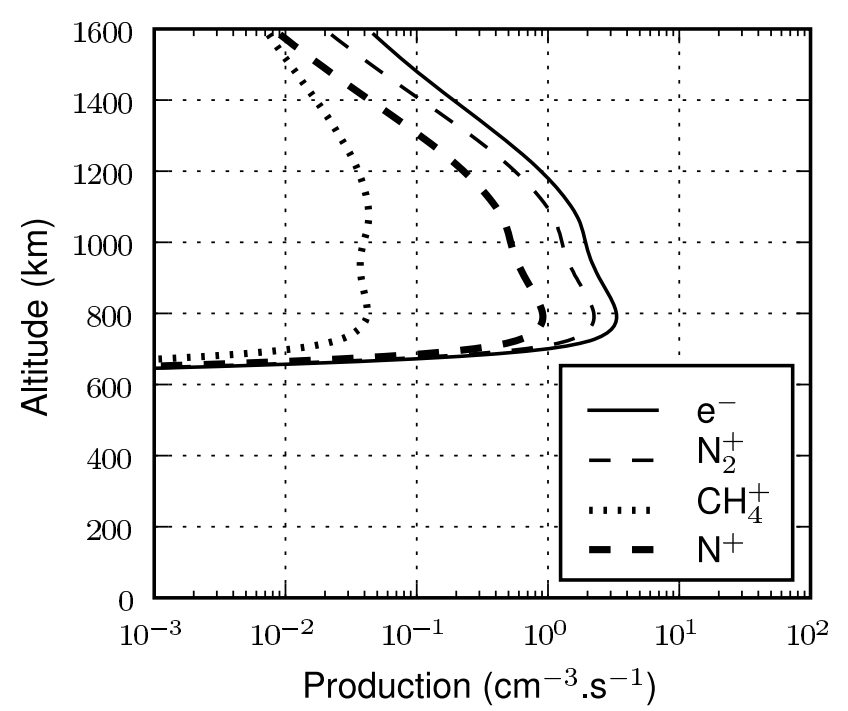

Fig. 4. Electron impact ionization in the (first) case of a vertical magnetic field line. $\mathrm{CH}_{4}^{+}$is produced by electron impact ionization on $\mathrm{CH}_{4}$. $\mathrm{N}_{2}^{+}$and $\mathrm{N}^{+}$are produced by electron impact ionization on $\mathrm{N}_{2}$. In this figure, the Müller-Wodarg et al. (2000) neutral atmosphere has been used because electrons reach the mesosphere, which is not described in the other neutral models.

of the primary flux plus the secondary electron flux) at different altitudes (Fig. 5, with the Cui et al. 2009, atmosphere in that case). We find a significant discrepancy between the Cassini flux measured at $1200 \mathrm{~km}$ and the model predictions. As in

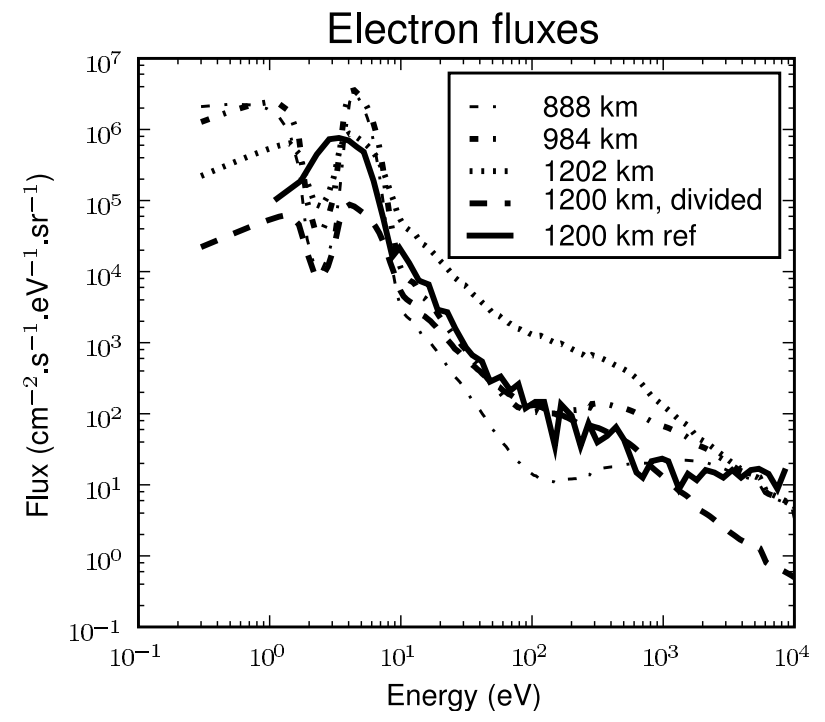

Fig. 5. Comparison of the modeled fluxes in the (first) vertical case at several altitude with $1200 \mathrm{~km}$ altitude Cassini measurements. This simulation shows that we cannot compare the vertical precipitation hypothesis with data. To compare with the result of the flux division hypothesis (Cravens et al. 2009), we added the results of that hypothesis, labeled "1200 km, ref".

Agren et al. (2007) and Cravens et al. (2009), we have to divide the input flux by a factor of about 10 to reach good agreement. As shown in Fig. 5, that hypothesis allows us to explain the flux in the 10-1000 eV energy range, but underestimates the flux at both lower and higher energies.

\subsection{Second case: straight line, horizontal at $1200 \mathrm{~km}$}

In that case, we use a straight horizontal line, whose lower altitude is $1200 \mathrm{~km}$, e.g., it is perpendicular to the vertical at this altitude. This geometry could not be simulated with the initial TransTitan code. The maximum altitude of this line is the top of our atmosphere at $1600 \mathrm{~km}$, and corresponds to the line with a $3500 \mathrm{~km}$ entry in the thermosphere and $0 \mathrm{~km}$ exit of the thermosphere, in Fig. 6. In that figure, we can see that the ionization peak is not at the middle of the line, but at $2300 \mathrm{~km}$, with an intensity of $0.3 \mathrm{~cm}^{-3} \mathrm{~s}^{-1}$. The corresponding altitude is $1230 \mathrm{~km}$, but is about $500 \mathrm{~km}$ away from the lowest point (1200 km altitude). The corresponding flux comparison can be seen in Fig. 7, the agreement being closer than in the previous case: the $1789 \mathrm{~km}$ length flux is inside the measurement error bars between $10 \mathrm{eV}$ and $200 \mathrm{eV}$, and corresponds to an altitude of $1200 \mathrm{~km}$. This results shows that the division of the input flux is unnecessary to account for this part of the spectrum. For the highest energy, our modeling departs from the measurements, being one order of magnitude higher in flux.

\subsection{Third case: straight line, horizontal at $1000 \mathrm{~km}$}

This case is similar to the previous one, except that the lower altitude of the line is $1000 \mathrm{~km}$ and the maximum altitude is always $1600 \mathrm{~km}$, which is reached at the extremities. The ion production maximum occurs at $3000 \mathrm{~km}$ length, with an intensity of $0.5 \mathrm{~cm}^{-3} \mathrm{~s}^{-1}$ (Fig. 8). The peak corresponds to an altitude of $1100 \mathrm{~km}$. The corresponding flux comparison can be seen in Fig. 9, where, fluxes at altitudes close to $1000 \mathrm{~km}$ show agreement with measurements when energies are above $500 \mathrm{eV}$. 


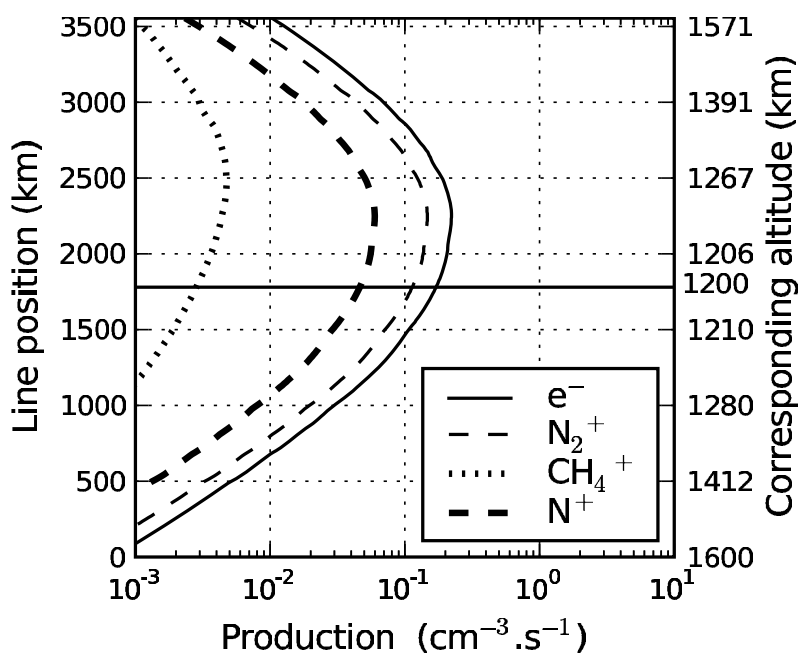

Fig. 6. Electron impact ionization in the (second) horizontal $1200 \mathrm{~km}$ case.

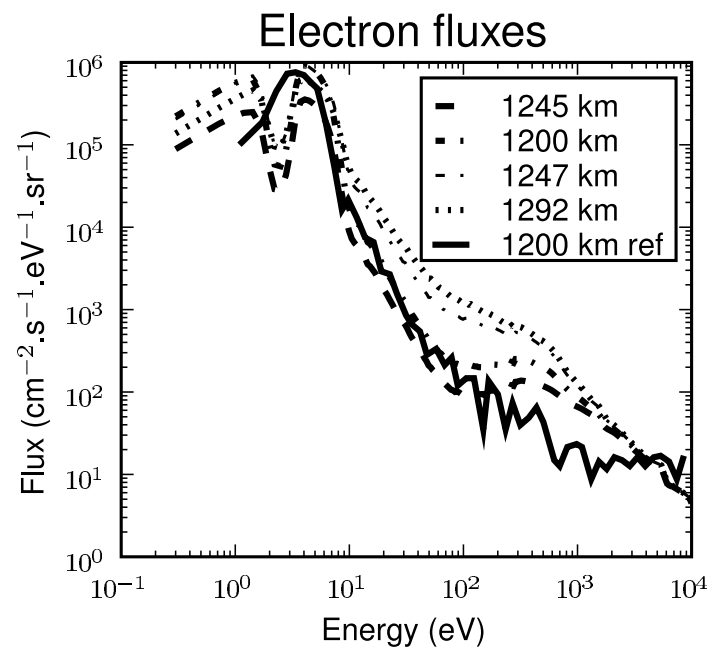

Fig. 7. Comparison of the fluxes at several altitude in the second case (horizontal, $1200 \mathrm{~km}$ ) with $1200 \mathrm{~km}$ altitude Cassini measurements. The altitude of $1245 \mathrm{~km}$ corresponds to an abscissa of $1192 \mathrm{~km}$. The $1200 \mathrm{~km}$ one to $1789 \mathrm{~km}$, the 1247 one to $2385 \mathrm{~km}$, and the 1292 one to $2624 \mathrm{~km}$. The higher the curvilinear abscissa is, the closer it is to the precipitation source.

\subsection{Fourth case: bent line, minimum at $1200 \mathrm{~km}$}

In this simulation, we use a curved line with a minimum altitude at $1200 \mathrm{~km}$ (Fig. 3). The ionization peak is at $1200 \mathrm{~km}$ length, which corresponds to an altitude of $1210 \mathrm{~km}$. Its intensity is $0.3 \mathrm{~cm}^{-3} \mathrm{~s}^{-1}$ (Fig. 12). The corresponding flux comparison can be seen in Fig. 11. A more accurate description of the reference flux is achieved for an altitude of $1300 \mathrm{~km}$, where the magnetic field line goes outside of the atmosphere.

\subsection{Fifth case: bent line, minimum at $1000 \mathrm{~km}$}

This is the same configuration as in the previous case, except that the lower altitude is $1000 \mathrm{~km}$. The ionization peak is at $1700 \mathrm{~km}$ length, which corresponds to an altitude of $1100 \mathrm{~km}$. Its intensity is $0.9 \mathrm{~cm}^{-3} \mathrm{~s}^{-1}$ (Fig. 12). The corresponding flux comparison can be seen in Fig. 13. The fluxes simulated below $1100 \mathrm{~km}$ are very close to the reference flux. For the two bent cases, we can conclude that it is possible to find a magnetic field line configuration

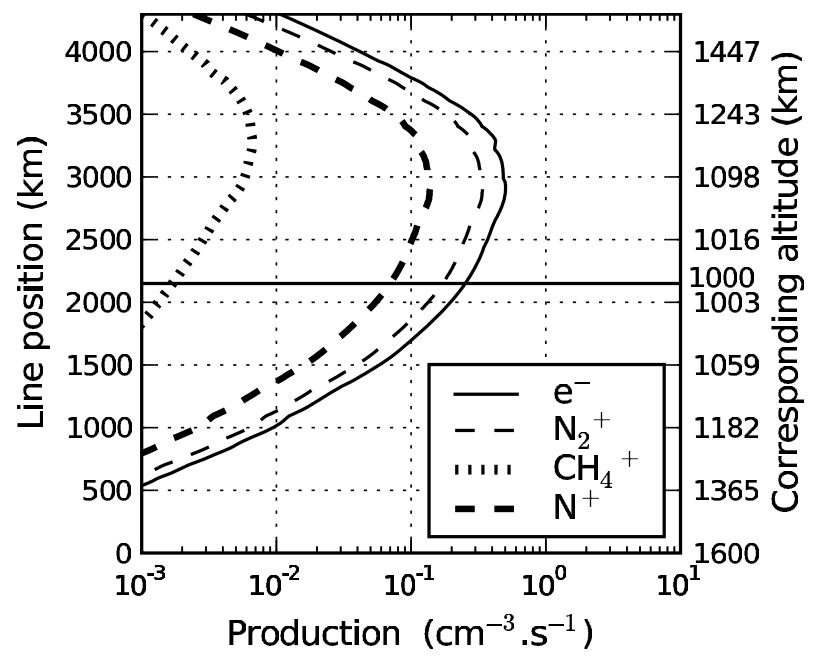

Fig. 8. Electron impact ionization in the (third) horizontal $1000 \mathrm{~km}$ case.

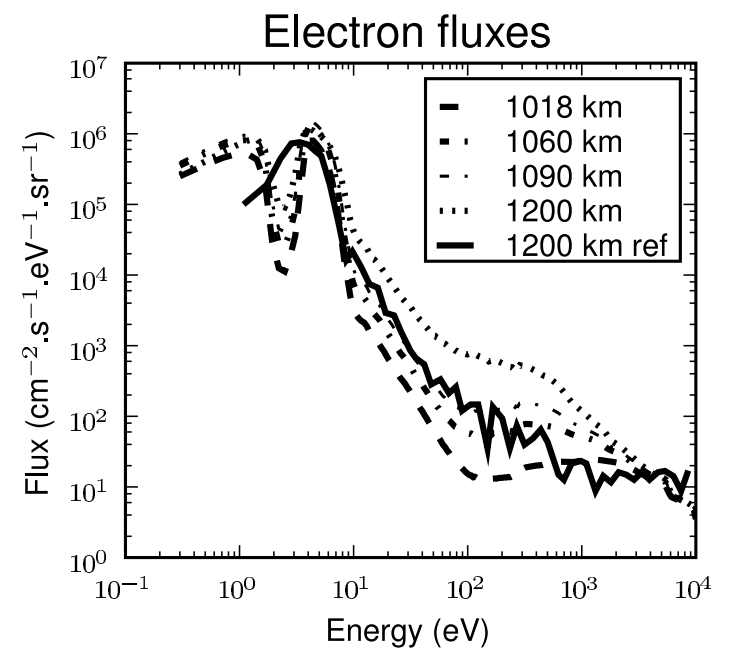

Fig. 9. Comparison of the fluxes at several altitudes in the third case (horizontal, $1000 \mathrm{~km}$ with $1200 \mathrm{~km}$ altitude Cassini measurements). The altitude of $1200 \mathrm{~km}$ corresponds to an abscissa of $3390 \mathrm{~km}$. The $1090 \mathrm{~km}$ one to $2957 \mathrm{~km}$, the 1060 one to $2813 \mathrm{~km}$, and the 1018 one to $2524 \mathrm{~km}$. The higher the curvilinear abscissa is, the closer it is to the precipitation source.

capable of explaining the entire spectrum without modifying the input energy spectrum. However, to determine the reality of this approach, we need to use global simulation computed lines.

\subsection{Sixth case: line from a hybrid simulation of the Ta fly-by of Cassini}

In this case, we use a magnetic field line computed from a hybrid simulation performed in the conditions of the Ta fly-by of Cassini (Modolo \& Chanteur 2008).

We considered several magnetic field lines in that simulation, and we only show the one that provide closer agreement with measurements. This line is of course an approximation, used as a representative field line when Titan is inside the magnetosphere of Saturn: the measurement were completed for the T5 fly-by and the MHD simulation is incomplete below $1400 \mathrm{~km}$. The ionization peak is at $500 \mathrm{~km}$ length, which corresponds to an altitude of $900 \mathrm{~km}$. Its intensity is $0.5 \mathrm{~cm}^{-3} \mathrm{~s}^{-1}$ (Fig. 14). The corresponding flux comparison can be seen in Fig. 15. The agreement 


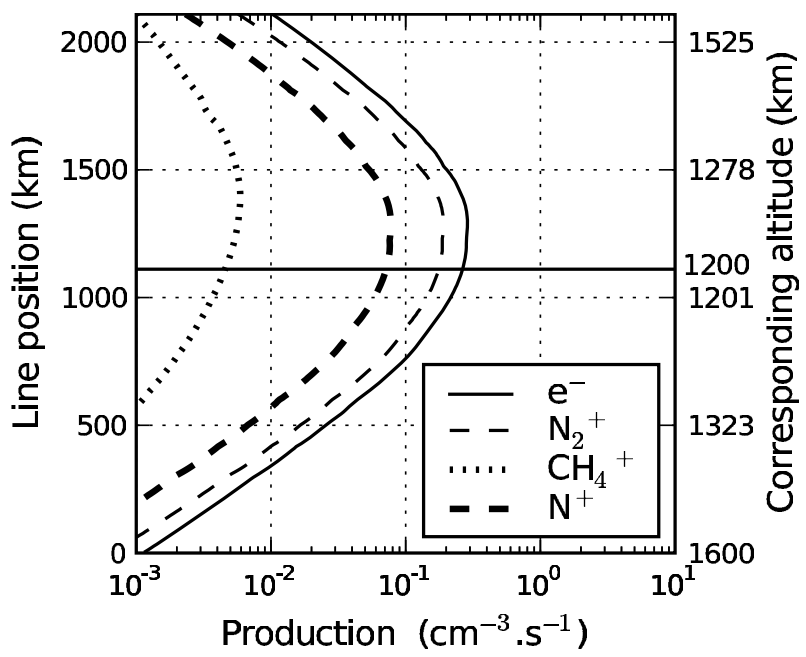

Fig. 10. Electron impact ionization in the (fourth) bent $1200 \mathrm{~km}$ case.

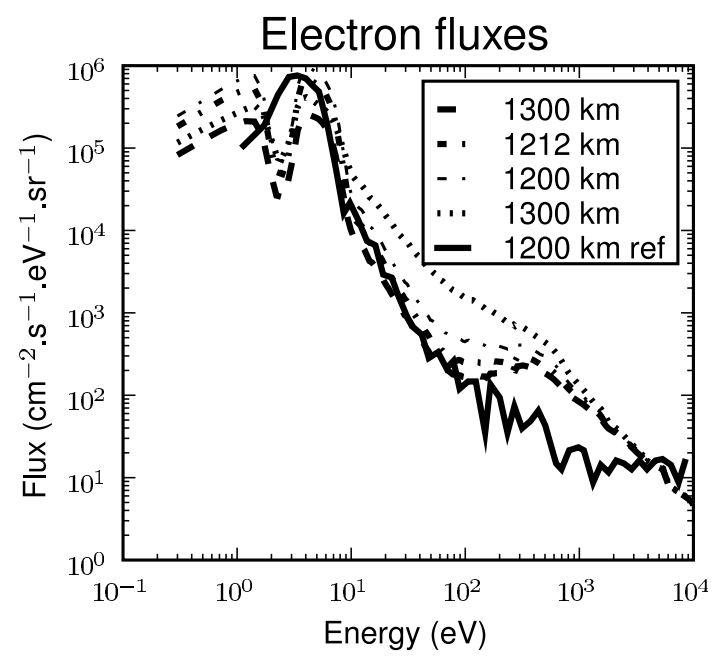

Fig. 11. Comparison of the fluxes at several curvilinear abscissa in the fourth case (bent, $1200 \mathrm{~km}$ ) with $1200 \mathrm{~km}$ altitude Cassini measurements. The altitude of $1300 \mathrm{~km}$ corresponds to an abscissa of $566 \mathrm{~km}$. The $1212 \mathrm{~km}$ one to $884 \mathrm{~km}$, the 1200 one to $1111 \mathrm{~km}$, and the 1300 one (dots) to $1557 \mathrm{~km}$. The higher the curvilinear abscissa is, the closer it is to the precipitation source.

between model and experiment is good and within the error bars in that case, even if a difference is still seen in high energies.

\section{Discussion}

Cravens et al. (2009) consider the problem of the discrepancy between the vertical precipitation model and the comparison of the fluxes at $1200 \mathrm{~km}$. In Fig. 5, we confirm this problem, with the important discrepancy between our model and the experiment at this altitude. The conclusion of this work was that the electrons are embedded in different magnetic tubes, each one having a different electron flux.

In the present work, the use of several magnetic field configurations shows that the geometry of the precipitation has an important effect on the electron flux at $1200 \mathrm{~km}$ altitude. In Figs. 7 and 15, we find good agreement between model and experiment, between approximatively $10 \mathrm{eV}$ and few hundred $\mathrm{eV}$. The differences at high energies could be explained by the spatial resolution: in Fig. 15, the high energy fluxes vary significantly between $1200 \mathrm{~km}$ altitude and $1130 \mathrm{~km}$ altitude. Another

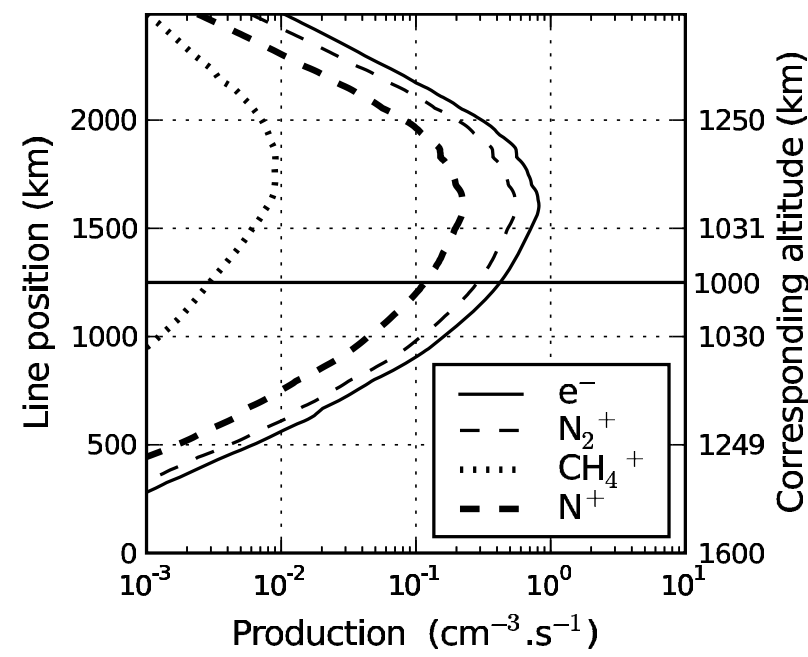

Fig. 12. Electron impact ionization in the (fifth) bent $1000 \mathrm{~km}$ case.

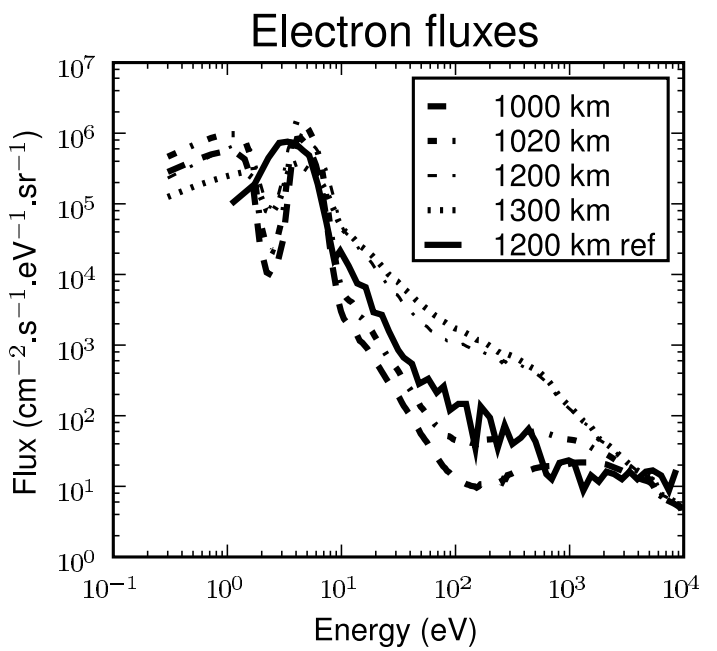

Fig. 13. Comparison of the fluxes at several curvilinear abscissa in the fifth case (bent, $1000 \mathrm{~km}$ ) with $1200 \mathrm{~km}$ altitude Cassini measurements. The altitude of $1000 \mathrm{~km}$ corresponds to an abscissa of $1253 \mathrm{~km}$. The $1020 \mathrm{~km}$ one to $1462 \mathrm{~km}$, the 1200 one to $1921 \mathrm{~km}$, and the 1300 one to $2088 \mathrm{~km}$. The higher the curvilinear abscissa is, the closer it is to the precipitation source.

explanation of this discrepancy could be that in the neutral atmosphere model used in our simulations, or in the uncertainties in the flux measurement at these energies. Concerning the influence of the neutral atmosphere, some minor differences in the fluxes are visible when we replace the Cui et al. (2009) model with either the Waite et al. (2005) or the Müller-Wodarg et al. (2000) model. However, the neutral atmosphere cannot notably explain the differences between model and experiment in the vertical case. In Fig. 16, we can see the resulting flux at $1200 \mathrm{~km}$ for the hybrid simulated line with the different neutral atmospheres. The neutral atmosphere of Cui et al. (2009) produces higher fluxes (about two times higher between 10 and $1000 \mathrm{eV}$ ). This is because of the lower density of that model, although the model predictions are within the error bars of the observations. Further improvements will require either a precise measurement of the neutral atmosphere during the fly-bys or a neutral atmosphere model that considers the variation in the density with e.g., solar zenith angle, solar activity.

To identify which of the two possible explanations of the observed electron flux is the most appropriate at $1200 \mathrm{~km}$, we 


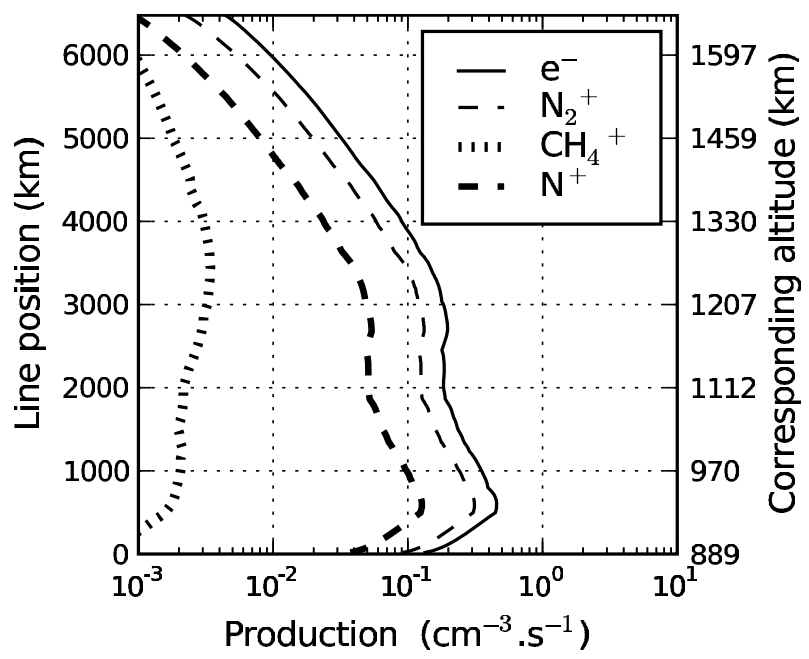

Fig. 14. Electron impact ionization in the Ta $1000 \mathrm{~km}$ case.

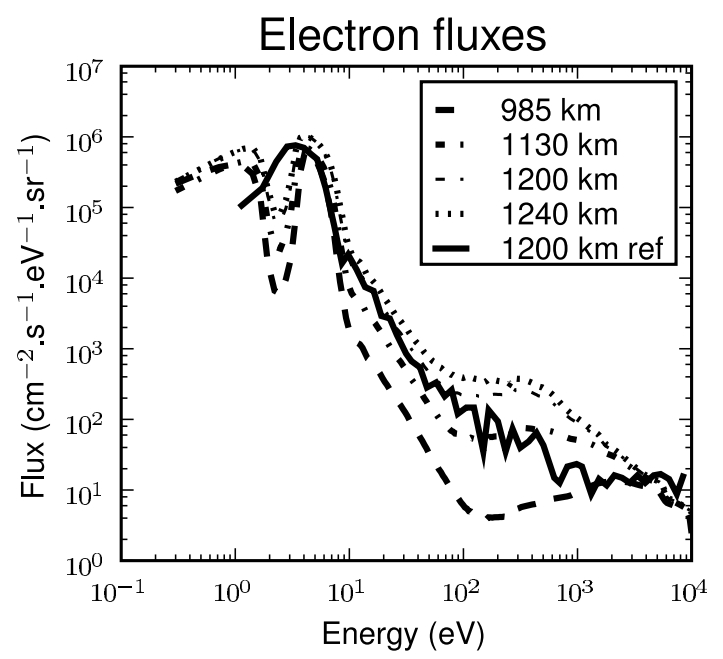

Fig. 15. Comparison of the fluxes at several curvilinear abscissa in the sixth case (Ta Fly-by) with $1200 \mathrm{~km}$ altitude Cassini measurements. The altitude of $985 \mathrm{~km}$ corresponds to an abscissa of $1087 \mathrm{~km}$. The $1130 \mathrm{~km}$ one to $2174 \mathrm{~km}$, the 1200 one to $2935 \mathrm{~km}$, and the 1240 one to $3262 \mathrm{~km}$. The higher the curvilinear abscissa is, the closer it is to the precipitation source.

need to measure the magnetic field line for the T5 conditions. Such work has not yet been published. The detection of a magnetic field oriented to the vertical at $45^{\circ}$ along the Cassini's path (Cravens et al. 2009) would be insufficient to retrieve the data by modeling, but would be able to constrain models. The observation of the magnetic draping (Bertucci et al. 2008) shows that an analysis of the magnetic environment of Titan is necessary to compute the ion production caused by electron impact. The ion production in the different cases (Figs. 7, 9, 11, 13, and 15) shows an enhancement in the production at high altitude, and a lack of production at low altitude.

\section{Conclusion}

The new version of TransTitan is able to compute electron precipitation along magnetic field lines in the atmosphere of Titan.

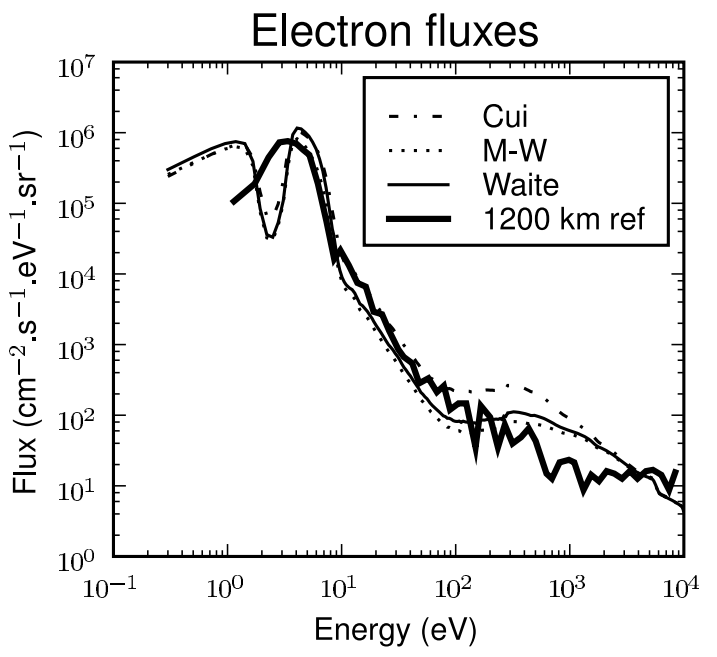

Fig. 16. Influence of the models of neutral atmosphere on the computed flux for the simulated line. All fluxes are computed at an altitude of $1200 \mathrm{~km}$ to be compared with the reference flux. The neutral atmosphere is not the main explanation of the difference at high energy, but needs to be considered for precise comparison of model and data (The Müller-Wodarg et al. 2000, atmosphere is labeled M-W).

Because of the magnetic field line geometry computed by hybrid simulation, we find agreement between model and observations of electron fluxes inside the atmosphere of Titan, without needing to divide the input flux. The magnetic field line geometry can therefore provide an explanation of the discrepancy between model and observation observed in Cravens et al. (2009), but lack of knowledge about the magnetic configuration still prevents us from being able to decide between our explanation and the hypothesis of magnetic tubes with different electron fluxes. The present work can be considered as a basis for future work on the comparison between MHD models and precipitation observations on Titan and other non-magnetic planets or bodies. It illustrates the importance of considering draped magnetic field lines inside the atmosphere.

Acknowledgements. The authors wish to thank C. Simon (BIRA, Belgium), M. Barthélemy (LPG, France) and V. Vuitton (LPG, France) for useful discussions.

\section{References}

Agren, K., Wahlund, J. E., Modolo, R., et al. 2007, Annales Geophysicae, 25, 2359

Backes, H., Neubauer, F. M., Dougherty, M. K., et al. 2005, Science, 308, 992 Bertucci, C., Achilleos, N., Dougherty, M. K., et al. 2008, Science, 321, 1475

Blanc, M., Kallenbach, R., \& Erkaev, N. V. 2005, Space Sci. Rev., 116, 227 Cravens, T. E., Robertson, I. P., Clark, J., et al. 2005, Geophys. Res. Lett., 32 Cravens, T. E., Robertson, I. P., Ledvina, S. A., et al. 2008, Geophys. Res. Lett., 35

Cravens, T. E., Robertson, I. P., Waite, J. H., et al. 2009, Icarus, 199, 174 Cui, J., Yelle, R. V., Vuitton, V., et al. 2009, Icarus, 200, 581

G. Gronoff, J. Lilensten, L. Desorgher, \& E. Flückiger, A\&A, 506, 955 (Paper I) Modolo, R., \& Chanteur, G. M. 2008, J. Geophys. Res., 113

Müller-Wodarg, I. C. F., Yelle, R. V., Mendillo, M., Young, L. A., \& Aylward, A. D. 2000, J. Geophys. Res., 105, 20833

Waite, J. H., Niemann, H., Yelle, R. V., et al. 2005, Science, 308, 982 